
Not for reproduction, distribution or commercial use.

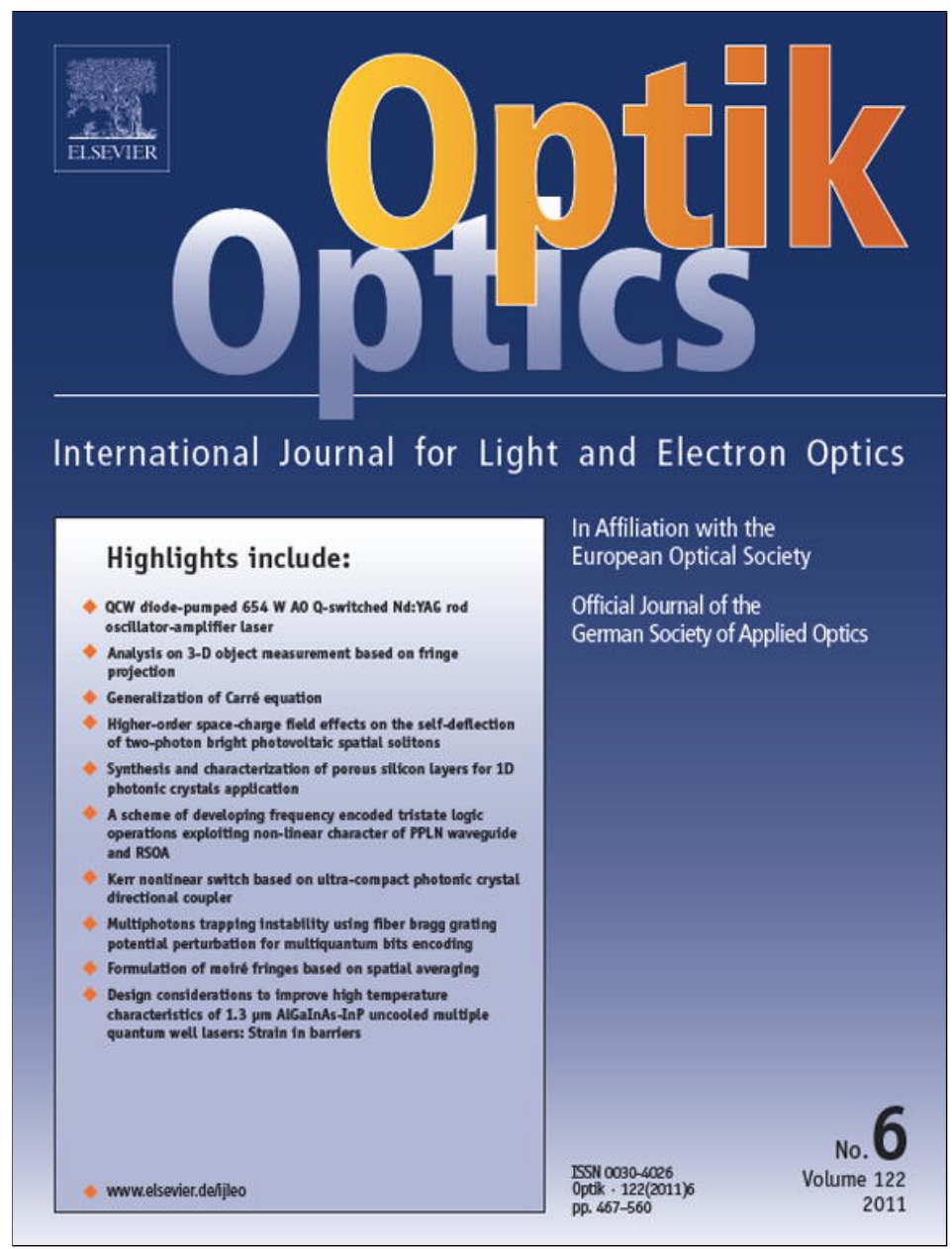

This article appeared in a journal published by Elsevier. The attached copy is furnished to the author for internal non-commercial research and education use, including for instruction at the authors institution and sharing with colleagues.

Other uses, including reproduction and distribution, or selling or licensing copies, or posting to personal, institutional or third party websites are prohibited.

In most cases authors are permitted to post their version of the article (e.g. in Word or Tex form) to their personal website or institutional repository. Authors requiring further information regarding Elsevier's archiving and manuscript policies are encouraged to visit:

http://www.elsevier.com/copyright 


\title{
Photorefraction images analysis through neural networks
}

\author{
Manuel F.M. Costa*, Sandra Franco, Mário R. Pereira \\ Universidade do Minho, Departamento de Física, 4710-057 Braga, Portugal
}

\section{A R T I C L E I N F O}

Article history:

Received 25 October 2009

Accepted 6 April 2010

\section{Keywords:}

Photorefraction

Visual screening

Infants

Image processing

Neural networks

\begin{abstract}
A B S T R A C T
The importance of an early evaluation of infants' visual system condition is long time recognized. Noncorrected visual disorders may lead to major vision and developmental non-reversible limitations in the future. Among the objective methods of refraction, photorefractive techniques are specifically designed for screening young children. Over the years a number of photorefraction systems with different grades of complexity and automation were developed. A critical problem that one needs to deal with in any approach to these systems is the interpretation and classification of the photorefraction images. In digital photorefraction conventional image processing operators and Fourier techniques were currently used. In this communication we will report on the use of Neural Networks for automated classification of digital photorefraction images.
\end{abstract}

(C) 2010 Elsevier GmbH. All rights reserved.

\section{Introduction}

Photorefraction is from the objective methods of refraction the most adequate for screening very young children. Essentially the method consists on delivering a light beam into the eyes. It is refracted by the ocular media, strikes the retina, focusing or not, reflects off and is collected by a camera. The sizes of the observed light patches vary with the defocus of each eye and that relates to the camera to subject's distance and to the pupil diameter. Qualitative or semi-quantitative information about refractive errors, accommodation, strabismus, amblyogenic factors and some pathology (e.g. cataracts) can be easily obtained.

Photorefraction can be implemented in three different approaches: orthogonal; isotropic; and eccentric photorefraction [1-7]. The differences arise basically from the type and arrangement of the optical components. Orthogonal and isotropic photorefractors have the flash source located along the optical axis of the camera lens, whereas in the eccentric method it is paraxial and eventually displaced outside the camera lens aperture. The light from a standard camera flash unit is delivered through a fiber optic. High aperture lenses should be used in order to capture the largest possible pointspread avoiding vignetting of the reflexes. Furthermore high focal lengths should be used (typically $50-500 \mathrm{~mm}$ ).

The use of video instead of photographic cameras has been reported allowing a much more convenient use of the photorefraction procedures. Orthogonal photorefractors feature a point light source centered in the camera lens and surrounded by four cylin-

\footnotetext{
* Corresponding author. Tel.: +351253 604060/4320; fax: +351253678981.

E-mail address: mfcosta@fisica.uminho.pt (M.F.M. Costa).
}

drical lens segments arranged at right angles in a star configuration. The acquired image from which measurements are made shows cross-centered on each eye. The length of the star arms indicates the defocus in two orthogonal directions. It increases with increasing defocus. However, in order to find the pupil diameter, at least one more photograph must be taken without the cylindrical lens segments in place.

The isotropic photorefraction is conducted using an apparatus identical to that used for orthogonal photorefraction but with no cylindrical lenses. Three photographs are taken while the subject looks in the direction of the camera with the camera's lens focused in the plane of the pupil to determine pupil size. The second and third photographs are taken with the camera focused at $50 \mathrm{~cm}$ and at $150 \mathrm{~cm}$, respectively, or $0.67 \mathrm{D}$ in front and behind the subject. In the myopic eye the picture focused at $50 \mathrm{~cm}$ will be more in focus than the other at $150 \mathrm{~cm}$. The opposite will be true of the hyperopic eye. By evaluating the relative diameter of the blur circles in the two out of focus pictures, refractive error can be assessed. When astigmatism is present a blurred ellipse is produced. When the subject's eye is myopically focused relative to the camera the blur circle is greater with the camera focused behind rather than in front of the subject. The opposite happens if the subject's eye is hypermetropic relative to the camera. This allows us to calculate the amount of hypermetropia or myopia present.

The main characteristic of eccentric photorefractors is that the flash source is placed off-centered. The camera is adjusted to provide a clearly focused image of the pupil to get the reddish reflex on the retina (as well as the first Purkinje reflex) [5,8]. If the subject's eyes are focused on the camera, light reflected will travel along its original path to the source. No light will be visible however in a subject for which the defocus of the eye exceeds a minimum amount that depends on the eccentricity of the flash source. When the 
threshold's refractive error is reached a crescent will first become visible in the pupil. The crescent appears on the flash's side for a myopic eye and conversely for hypermetropic ones. Controlling the eccentricity, focal distance of the camera's lens and object to camera distance the sensitivity of the method can be controlled.

For young children a high operation distances is highly recommended and thus higher eccentricity and focal distances should be used. Fortunately infants' pupils are usually wide open, which do help the test! For other kind of subjects smaller camera to subject distances may be used.

The detection of errors exceeding $0.15 \mathrm{D}$ can be attained. The detection of astigmatism can be done placing the light source at different orthogonal positions around the camera's optical axis.

\section{Materials and methods}

Different implementation of the eccentric photorefraction method exist and are even commercially available. At the Optometry and Vision Sciences Group of University of Minho' Center of Physics the method was implemented in two different approaches: one main system technologically more complex, accurate, robust and versatile; and a portable one. In both cases two of the different methods of photorefraction, the isotropic and the eccentric, can be used.

The main system (Fig. 1) is essentially formed by one CCD color camera (Cohu' 2200 series) connected to a microcomputer through a Matrox Corona frame grabber, and a light source (white flash light source) coupled to one or two optical fibers (color filters, red and green, are placed at the entrance of the fiber, placed orthogonally one to the other, allowing simultaneous recording of a two-inone image, thus allowing a fast and more accurate measure of the patients' eyes astigmatism). The fiber optics ( $1 \mathrm{~mm}$ diameter plastic fiber) is placed eccentrically or not, depending on the method, in front a teleobjective (Sigma 60-300).

The eccentricity can be controlled by a step motor driven $\mathrm{XYZ}$ stage. A simple electronic circuit using the strobe output synchronization signals of the Corona frame grabber makes the synchronization of the flash to the image grabbing. Even so there is a percentage of around 30\% of fault images acquired. By that reason not only one image is taken each time but series of 10 images, taking advantage of the on board internal memory. This turns to be also very convenient with the most reluctant or active children. A routine written on the Matrox Inspector image processing takes care of the whole image acquisition and processing tasks. The output of the program is a series of result' tables and images, including the originally acquired in MPEG format (allowing the operator to fol-

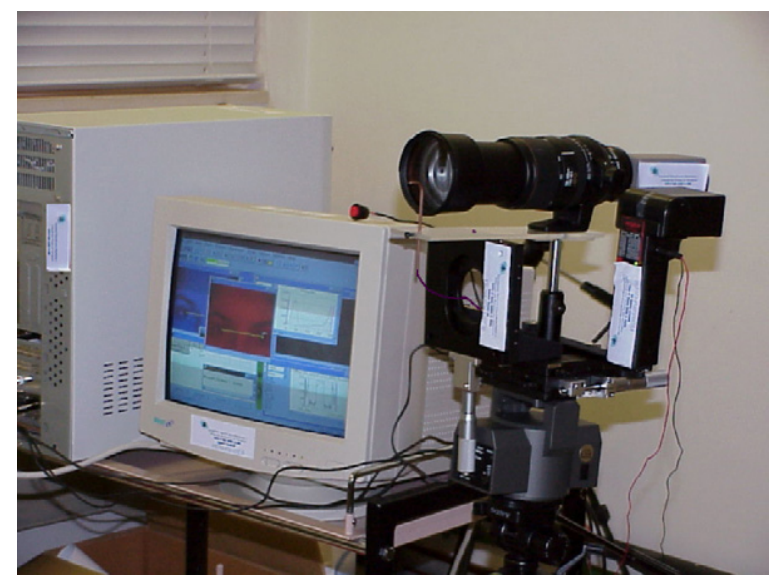

Fig. 1. The main photorefraction system. low different image processing strategies). A database is actualized for each inspection process.

During the development of the test the camera's image is digitized and displayed in real time. After digitizing the eye's images are processed in less than $5 \mathrm{~s}$. A number of basic processing techniques are applied together with some more advanced ones like blob counting and measuring and pattern matching. The image processing techniques include: histogram equalization and contrast enhancement, noise reducing by low pass Fourier filtering; edge enhancement and location by the application of differential operators, posterization or binarization; color channel splitting, histogram analysis, and profile sectioning, blob analysis, matching and feature extraction [8].

This system is to be used with the child comfortably seat on the parents' lap at distances ranging from three to five meters away from the system.

The portable system version is a really simple and straightforward solution. One just take profit of new developments on digital photographic cameras. They have excellent prices for the optical quality and image resolution offered. Furthermore the issue of flash synchronization is already solved. The major problem is the focal distance and the lens aperture offered that precludes its use at comfortable distances and vignettes the crescents formed. At much higher prices there are cameras that allow the substitution of the objective by standard teleobjectives.

However for instance the Sony Digital Mavica FD91 digital camera presents already a suitable objective both in terms of zoom and aperture. A plastic optical fiber was coupled to the flash output and fix the other end in a small stand in from the objective that allows an easy manual control of the illumination eccentricity. High-resolution XGA images are obtained of patients' eyes at distances between 2 and $4 \mathrm{~m}$.

The major drawback is that no direct connection to a computer is possible. However, this ads portability to the setup (in most situations the autofocus function of the camera can be used) that can be taken to any place allowing the acquisition of images even without previous children preparation. The acquired images are taken to microcomputer, using conventional diskettes, and processed the same way as the ones of the main system in equivalent routines.

Traditionally the processed images can be, directly and immediately after the acquisition, analyzed by the operator in an easier fast and more efficient way. The relevant images and data files are suitably compressed and stored on patient's files for future reference. With the photorefraction setup the operator, optometrist or ophthalmologist, is able to acquire the number of different images that each particular situation demand. Furthermore this can be made very quickly reducing to a minimum the stress the child may feel. Qualitative and quantitative information about refractive errors, accommodation, strabismus, amblyogenic factors or even some pathology (cataracts) can be easily obtained. Refractive errors between one and three diopter can be estimated with a resolution down to $0.25 \mathrm{D}$. Pupil's diameter for anisocoria evaluation and interpupilary distances can be measured with sub-millimeter resolution.

Artificial neural networks (NNs) are dynamic systems that are conformed of a number of processing units with weighted connections to each other [9-12]. The output of a unit determines its level of activity while the weight value of the connection between two units determines the strength of the connection. Each unit receives a number of inputs from other units and after processing, sends its output to other units through the weighted connections. The neural network as a whole acts by designating some units to receive their input signals from the environment and others to send their output signals back to the environment. A number of units that will have no contact with the environment are designated as hidden units. 
An NN reacts dynamically to an external input returning an output that can be viewed as the behavior that emerges from the interactions of the large number of processing units. Useful computational tasks can thus be performed even with very high levels of complexity in an effective and automated way. One of the most important characteristics of NNs is the ability to perform, define and redefine the computational tasks by learning from examples and even generalizing to new situations. In practical applications, training examples are usually measurements and observations from the physical process, itself, to be modeled.

The general principle of learning algorithms is applicable to any NN architecture. It consists in establishing the arrangement and pattern of connectivity of the elements of the network (the neurons).

Using the training data the NN will find a set of weights such that the network behaves in a desired fashion. This is achieved by initially assigning small random values to the weights and gradually adjusting them according to the adjust/matching of the NN outputs to the measured data of the training examples. The network weights are modified to reduce the total network error.

Back propagation is the most popular supervised learning scheme. Each learning step of the algorithm consists of two phases: a forward phase in which an input value, vector or matrix from the training set is presented to the network and the activation state of the entire network is determined; and a backward phase in which the target output vector is used to determine the contribution of each processing unit to the total network error. The process will be repeated with the corrected weights until the network reaches a defined minimum. This procedure is repeated for all training vectors sequentially or randomly until the network error converges to an acceptable level.

Despite the fact that training data may be fuzzy or corrupted with noise, the NNs are robust enough to be able to extract the underlying structure from the data and thus provide an implicit representation of the physical process being modeled. And that's one of the main reasons why we decided to apply NN to the classification of our noisy, low contrast, photorefraction images.

In order to discriminate between the different situations we simply have to look and distinguish the level of illumination and eventually the size of crescent on the eye's pupil.

\section{Results}

In order to illustrate the type of images our NN will deal with a set of representative images obtained with our eccentric photorefractor is presented bellow (Figs. 3-5).

In Fig. 3 the image of the normal eye of a 8-year old child, obtained with the portable photorefraction system (Fig. 2), is presented. The measured diameter pupil 5.5 and $5.1 \mathrm{~mm}$ and areas

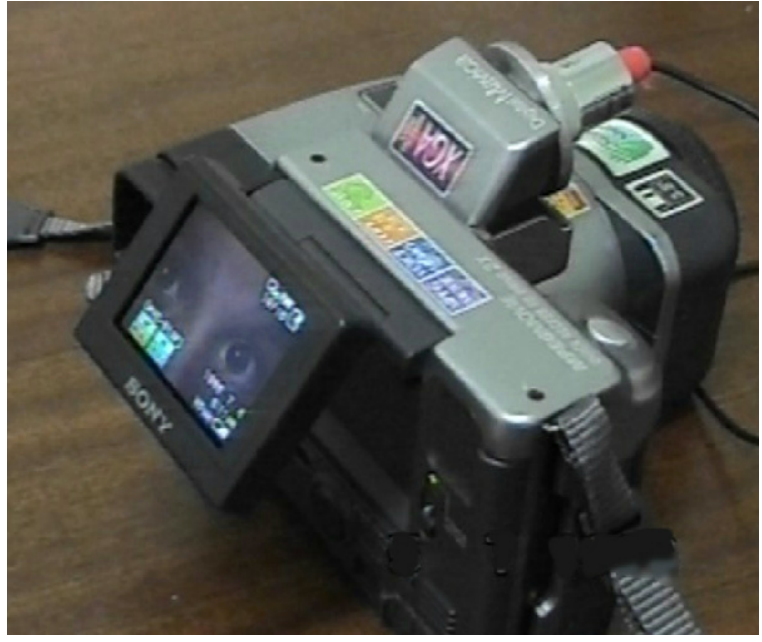

Fig. 2. The portable photorefractor showing the acquired image used for illustrating the processing process (Figs. 3-5).

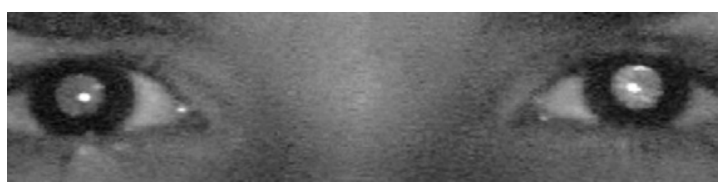

Fig. 3. The photorefraction image of a normal eye.

( $33 \mathrm{~mm}^{2}$ on the right eye and $28 \mathrm{~mm}^{2}$ on the left eye); are computed as well as the interpupilary distance $(61 \mathrm{~mm})$. In Fig. 4 we isolated the pupil area from the first Purkinje reflection making the visual evaluation process very easy.

Two NN were established. The simpler one had as task just to decide if the photorefraction images are from peoples with no major refractive error or if a significant refractive error (above 1 diopters, spherical or astigmatic) exists.

The acquired images have typically 16,384 pixels corresponding to 64 rows and 256 columns, with a gray level depth of 8 bit (256 levels).

In order to improve speed and reduce our images' noise, the number of inputs had to be reduced to reasonable values. We concluded that it was acceptable (Fig. 5) in terms of efficiency and processing speed to use 2 [8] input cells (64 per 16 pixels) [13].

On the basic NN only 16 hidden cells were established and just two binary output cells (one corresponding to the status of each eye).

On the advanced NN we would like to discriminate between eyes with refractive errors (both spherical and cylindrical) on the levels -5 to +5 diopters, on half diopter steps.
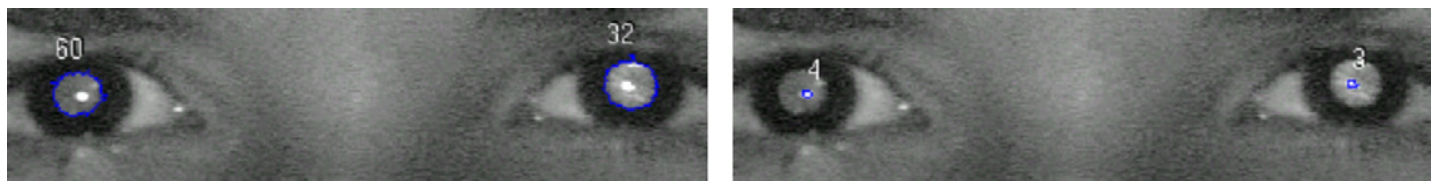

Fig. 4. Isolation of the pupil's area (left), and, automatic identification of the Purkinje reflex (right).
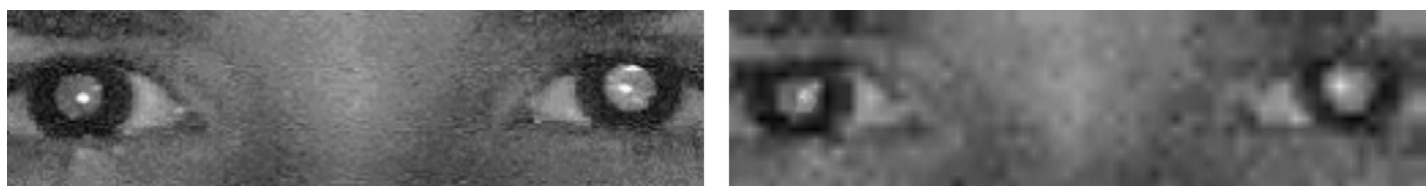

Fig. 5. In order to improve the speed the NN application and reduce our image's noise, we proceed with a dimensionality reduction of our input matrix data. All relevant features are still available allowing an error free distinction between eyes with or without refractive problems (right' photo). 


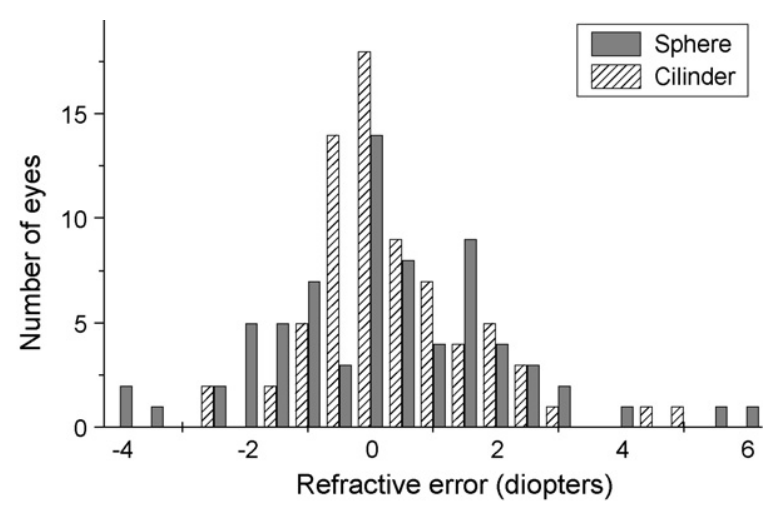

Fig. 6. Refractive error distribution of our training samples.

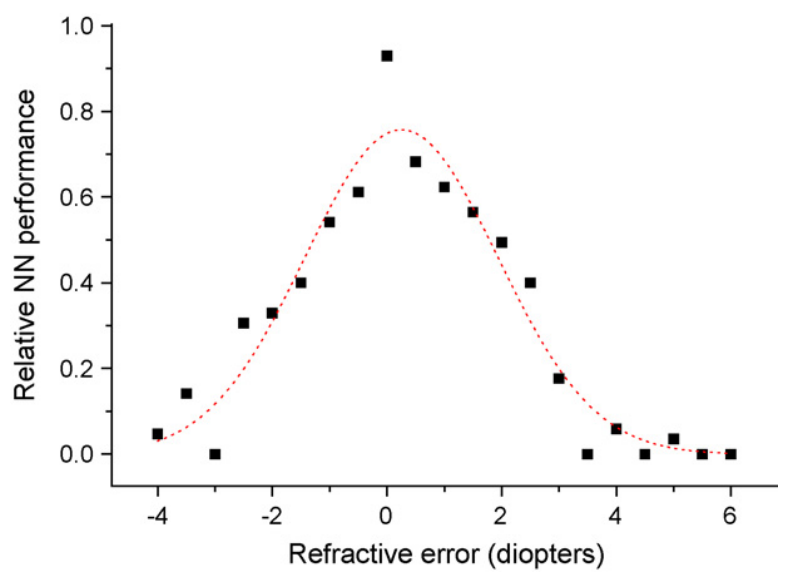

Fig. 7. Plot of the performance of the advanced NN versus the spherical refractive error. The dotted line was obtained by Gaussian fitting of the data.

The number of inputs rose to 4096 . As a first processing stage we simply discarded the input cells corresponding to the two middle quarters of the original image $(64 \times 256$ pixels $)$. We thus will have an input layer with 2048 cells. Based on the number of input cell and its organization on rows and columns, but also on a trial and error approach, we established a hidden layer with 72 neurons. The output layer has five units.

The same data was used for the training of both neural networks. A set of 72 eye images were used. On the graph of figure we show the distribution of those eyes into different classes of refractive error both spherical and astigmatic.

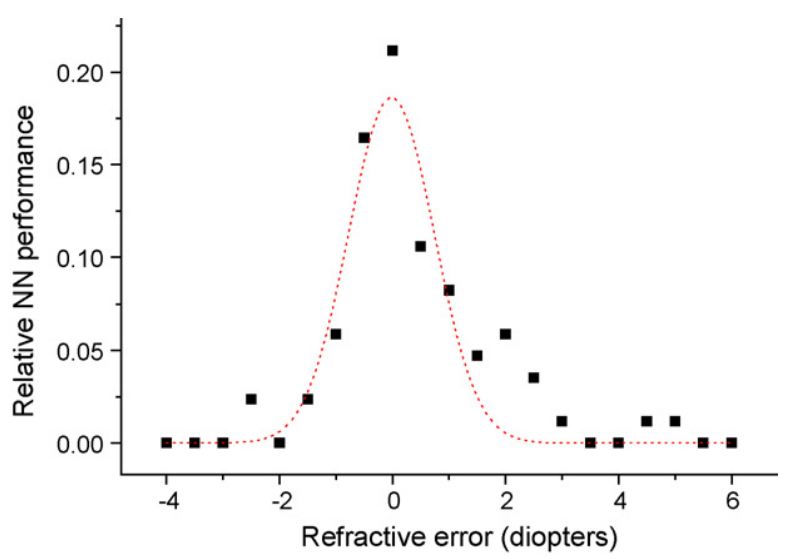

Fig. 8. Plot of the performance of the advanced NN versus the cylindrical refractive error. The dotted line was obtained by Gaussian fitting of the data.
Almost 17\% are myopically "good" eyes (refractive error less than \pm 0.5 diopters). When considering the classification of our basic NN parameter only $53 \%$ eyes present significant refractive spherical errors. In what concerns astigmatism $25 \%$ (or $71 \%$ with the criteria of the basic NN) no significative refractive error (Fig. 6.).

The number of training samples was more then enough for the basic NN and thus a $100 \%$ classification is attained until now. In what concerns the advanced $\mathrm{NN}$ the network performance depends on the level of refractive error. Results of the performance of the advanced NN obtained with over 100 patient's images are shown in Figs. 7 and 8.

\section{Conclusion}

The incorporation of new rapidly developing technologies as digital cameras, fiber optics, optical imaging and image processing, on new instruments and sensors is extremely important for the improvement of medical diagnosis in a number of different fields. Our photorefractors can be considered as a useful equipment for the visual screening of infants.

Artificial neural networks are a very convenient tool for classification of the photorefraction images not only for the simple detection of refractive problems but also for its classification into commonly accepted deficiency level.

\section{Acknowledgement}

The authors would like to acknowledge Dr. J. Jorge for his contribution in the process of setting up the main photorefraction system of the Optometry and Vision Sciences Group of University of Minho' Center of Physics in a work supported by the "Secretariado Nacional para a Reabilitação" in the frames of the program CITE III.

\section{References}

[1] R. Duckman, B. Meyer, Use of photoretinoscopy as a screening technique in the assessment of anisometropia and significant refractive error in infants/toddlers/children and special populations, Am. J. Optom. Physiol. Optic. 64 (1987) 614-620

[2] H.C. Howland, Optics of photoretinoscopy: results from ray tracing, Am. J. Optom. Physiol. Optic. 62 (1985) 621-625.

[3] W.R. Bobier, O.J. Braddick, Eccentric photorefraction: optical analysis and empirical measures, Am. J. Optom. Physiol. Optic. 62 (1985) 614-620.

[4] W.R. Bobier, Quantitative photorefraction using an off-center flash source, Am. J. Optom. Physiol. Optic. 65 (12) (1988) 962-971.

[5] H.C. Howland, N. Sayles, C. Cacciotti, M. Howland, Simple pointspread retinoscope suitable for vision screening, Am. J. Optom. Physiol. Optic. 64(1987) $114-122$.

[6] F. Schaeffel, L. Farkas, H.C. Howland, Infrared photoretinoscope, Appl. Opt. 26 (1987) 1505-1509.

[7] M.F.M. Costa, J.M.M. Jorge, Digital photorefraction, in: Proceedings of the International Conference “European Biomedical Optics Week-BiOS Europe’97, San Remo, Italia, September 1997, Proc. Soc. Photo-Opt. Instrum. Eng. 3199 (1998) 13-21.

[8] M.F.M. Costa, S. Franco, Improving contact lens' fitting evaluation by the application of image processing techniques, Int. Contact Lens Clin. 25 (1) (1998) 22-32.

[9] D.E. Rumelhart, D. Richard, G. Richard, Y. Chauvin, Back-propagation: Theoretical Foundations, Edited from Back-propagation and Theory, lst edn., Lawrence Erlbaum, 1992

[10] R.D. Rumelhart, J. Mc-Clelland, Parallel Distributed Processing, lst edn., MIT Press, Cambrídge, MA, 1986.

[11] G. Hinton, Connections learning procedures, Artifi. Intell. 40 (1989) 185-234.

[12] R.D. Payne, M.A. Matteson, A.L. Moran, Application of neural networks in spray forming technology, Int. J. Powder Metall. 29 (4) (1993) 345-351.

[13] M.F.M. Costa, Automated analysis of electrophoretic gels by image digitalization and processing, Imaging Sci. J. 48 (2001) 177. 